\title{
Equation of State Behavior and Interfacial Properties of Lattice Chain Fluids: A Comparison of Lattice Fluid Theory and Monte Carlo Simulation
}

Citation for published version (APA):

Nies, E. L. F., \& Cifra, P. (1994). Equation of State Behavior and Interfacial Properties of Lattice Chain Fluids: A Comparison of Lattice Fluid Theory and Monte Carlo Simulation. Macromolecules, 27(21), 6033-6039. https://doi.org/10.1021/ma00099a015

DOI:

10.1021/ma00099a015

Document status and date:

Published: 01/01/1994

Document Version:

Publisher's PDF, also known as Version of Record (includes final page, issue and volume numbers)

\section{Please check the document version of this publication:}

- A submitted manuscript is the version of the article upon submission and before peer-review. There can be important differences between the submitted version and the official published version of record. People interested in the research are advised to contact the author for the final version of the publication, or visit the DOI to the publisher's website.

- The final author version and the galley proof are versions of the publication after peer review.

- The final published version features the final layout of the paper including the volume, issue and page numbers.

Link to publication

\footnotetext{
General rights

- You may freely distribute the URL identifying the publication in the public portal. follow below link for the End User Agreement:

www.tue.nl/taverne

\section{Take down policy}

If you believe that this document breaches copyright please contact us at:

openaccess@tue.nl

providing details and we will investigate your claim.
}

Copyright and moral rights for the publications made accessible in the public portal are retained by the authors and/or other copyright owners and it is a condition of accessing publications that users recognise and abide by the legal requirements associated with these rights.

- Users may download and print one copy of any publication from the public portal for the purpose of private study or research.

- You may not further distribute the material or use it for any profit-making activity or commercial gain

If the publication is distributed under the terms of Article $25 \mathrm{fa}$ of the Dutch Copyright Act, indicated by the "Taverne" license above, please 


\title{
Equation of State Behavior and Interfacial Properties of Lattice Chain Fluids: A Comparison of Lattice Fluid Theory and Monte Carlo Simulation
}

\author{
Erik Nies* and Peter Cifra ${ }^{\dagger}$ \\ Centre for Polymers and Composites, Department of Polymer Technology, \\ Eindhoven University of Technology, 5600 MB Eindhoven, The Netherlands
}

Received July 22, 1993; Revised Manuscript Received March 28, $1994^{\circ}$

\begin{abstract}
Monte Carlo simulation results for interacting chains at zero and elevated pressures are presented using a new simulation method. Both bulk and interfacial properties are investigated over a broad range of pressures and temperatures, thus covering high and low lattice fluid densities. The bulk simulation data are used to investigate the predictive capabilities of the Sanchez-Lacombe lattice fluid theory and an equation of state theory including nonrandom effects which are assessed using Guggenheim's quasichemical approximation. The nonrandom mixing theory accurately predicts all available data even up to the lowest densities whereas the Sanchez-Lacombe theory overestimates moderate densities and completely fails at low densities. Interface profiles exhibit generally larger density variations for the systems including interactions in comparison to athermals, and the effect of pressure is concentrated in the system on the interface.
\end{abstract}

\section{Introduction}

In the present contribution the equation of state and interfacial properties of the classical lattice model of chain molecules are investigated by Monte Carlo (MC) simulations and compared to theoretical results. The evaluation of the thermal properties of polymer systems is frequently done by using an equation of state theory. A number of such theories are available in the literature which are more or less successful in the description and prediction of experimental data. ${ }^{1-9}$ However, in many instances deviations remain between experiment and theory and often the origin of the discrepancies is not really clear. Certainly, the adapted molecular model is an important cause for deviations, but also the analytical theory is in error due to the inevitable approximations. With the advance of computer simulations these latter approximations can be investigated systematically, since simulation and theory can be compared for a molecular model of interest. There are relatively few $\mathrm{MC}$ studies on polymers which consider variations in pressure. .0-12 $^{12}$ This is especially true for the simulation of the condensed polymer phase of sufficiently long polymer chains with interactions. Here the volume fluctuations necessary to study the pressure dependence are difficult to employ due to entanglements.

\section{Theory}

The lattice model at hand consists of $N s$-mers, each occupying $s$ consecutive sites on a partially filled lattice with coordination number $z$, volume $V$, and temperature $T$. Furthermore, pairs of nonbonded nearest-neighbor mers are characterized by a contact energy $-\epsilon$. The volume of the system and the lattice filling fraction $y$ are given by

$$
V=\left(s N+N_{h}\right) \mathrm{v}_{0}
$$

and

$$
y=\frac{s N}{s N+N_{h}}=\frac{s N \mathrm{v}_{0}}{\left(s N+N_{h}\right) \mathrm{v}_{0}}=\frac{1}{\tilde{V}}=\tilde{\rho}
$$

\footnotetext{
† Permanent address: Polymer Institute, Slovak Academy of Sciences, 84236 Bratislava, Slovakia.

- Abstract published in Advance ACS Abstracts, August 1, 1994.
}

where $N_{\mathrm{h}}$ is the number of vacant lattice sites and $\mathrm{v}_{0}$ is the volume of a lattice site. The last equality relates the filling fraction to the reduced or scaled volume $\tilde{V}$ and density $\rho$.

Other useful variables pertinent to the subsequent discussion are summarized and explained as follows: the nonbonded contacts of an $s$-mer $z q_{\mathrm{c}}=s(z-2)+2=s z(1$ $-\alpha)$ with $\alpha=\gamma(1-1 / s)$ and $\gamma=2 / z$; the nonbonded contact fraction $q=z q_{\mathrm{c}} N /\left(z q_{\mathrm{c}} N+z N_{\mathrm{h}}\right)=(1-\alpha) y /(1-\alpha y)$; the total number of segmental contact pairs $Q=z q_{\mathrm{c}} N / 2$; the number of segment-vacancy contact pairs $Q x$, with $x$ a microscopic parameter defining the fraction of segmentvacancy contact pairs in relation to the total number of segmental contact pairs $Q$. It is shown below that in the case of random mixing the parameter $x$ becomes

$$
x=x^{*}=q(1-q)
$$

The equation of state can be derived from the Helmholtz free energy $A$

$$
p=-\partial A /\left.\partial V\right|_{N, T}
$$

According to statistical mechanics, the configurational part of the free energy is defined by ${ }^{13}$

$$
A=-k T \ln \left(Z_{N}\right)
$$

and the configurational partition function $Z_{N}$ can be calculated, using ${ }^{14}$

$Z_{N}(N, T, V)=\sum_{\{x\}} g(N, T, V, x) \exp \left[-\frac{E_{0}(N, T, V, x)}{k T}\right]$

where $E_{0}(N, T, V, x)$ is the internal energy and $g(N, T, V, x)$ is the number of configurations available to the system under the constraints of volume, temperature, and number of segment-hole contacts. The sum in eq 6 extends over all possible values of $x$ in agreement with the required $N$, $V$, and $T$.

The interaction energy can be expressed as the product of the pair interaction energy $-\epsilon$ and the number of contacts between segments

$$
\frac{E(N, T, V, x)}{k T}=-s N \frac{1-\alpha}{\gamma}\left(\frac{q-x}{q}\right)\left(\frac{\epsilon}{k T}\right)
$$


If random mixing is assumed, the internal energy becomes

$$
\frac{E(N, T, V)}{k T}=-s N \frac{1-\alpha}{\gamma} q\left(\frac{\epsilon}{k T}\right)
$$

In eqs 7 and 8 proper account is taken of the effective nonbonded contacts of the $s$-mers. If one allows the coordination number $z$ to approach infinity $(z \rightarrow \infty)$, the classical van Laar-Hildebrand expression is recovered with $\epsilon^{*}=z \epsilon / 2$ finite.

$$
\frac{E(N, T, V)}{k T}=-s N y\left(\frac{z \epsilon}{2 k T}\right)=-s N y\left(\frac{\epsilon^{*}}{k T}\right)
$$

The number of configurations $g(N, T, V)$ is approximated by $y^{9,14}$

$$
\begin{array}{r}
\ln [g(N, T, V, x)]=\ln \left(N_{H}\right)-\frac{1-\alpha}{\gamma q}[2 q \ln (q)+ \\
2(1-q) \ln (1-q)-(q-x) \ln (q-x)-2 x \ln (x)- \\
(1-q-x) \ln (1-q-x)]
\end{array}
$$

and

$$
\begin{aligned}
\ln \left(N_{H}\right)=-\frac{\ln (y)}{s}-\frac{1-y}{y} \ln (1-y)+ & \\
& \frac{1-\alpha y}{\gamma y} \ln (1-\alpha y)
\end{aligned}
$$

The bracketed term in eq 10 represents the influence of the nonrandom mixing of segments. In the case of random mixing, this contribution vanishes and the random combinatorial entropy, given by the normalization factor $N_{H}$, is obtained. In the present theory the combinatorial entropy originally derived by Huggins ${ }^{15}$ is recovered. In comparison with the well-known Flory ${ }^{16}$ result, the Huggins equation contains an extra contribution, i.e., the last term in eq 11, representing the influence on the combinatorial entropy of the segmental connectivity. This latter correction vanishes when the coordination number $z \rightarrow \infty$. More recently, Freed ${ }^{17}$ has presented an expansion of the exact lattice entropy introducing systematic corrections to the Flory result. Although the Huggins expression does not present one or more of these systematic corrections, in practice it constitutes the major correction to the Flory result in comparison with the exact entropy known from lattice simulations for athermal systems. It is customary to approximate the partition function by the maximum term of the sum in eq $6^{14}$

$Z_{N}(N, T, V) \simeq g(N, T, V, \bar{x}) \exp \left[-\frac{E_{0}(N, T, V, \bar{x})}{k T}\right]$

where $\bar{x}$ is the value of $x$ for the most probably configuration and can be calculated from the so-called quasi-chemical (QC) equation.

$$
\left.\frac{\partial Z_{N}}{\partial x}\right|_{N, T, V}=\mathrm{e}^{\epsilon / k T}-\frac{\bar{x}^{2}}{(1-q-\bar{x})(q-\bar{x})}=0
$$

This extremum condition (13) can be solved for $\bar{x}$

$$
\bar{x}=\frac{1-\left[\left(1-4 q(1-q)\left(1-e^{t / k T}\right)\right]^{1 / 2}\right.}{2\left(1-e^{\varepsilon / k T}\right.}
$$

As anticipated in the beginning, for athermal systems $(-\epsilon)$ $k T=0$ ) the random mixing value for $x$ is obtained

$$
x^{*}=\lim _{\epsilon \rightarrow 0}\left[\frac{1-\left[\left(1-4 q(1-q)\left(1-\mathrm{e}^{\epsilon / k T}\right)\right]^{1 / 2}\right.}{2\left(1-\mathrm{e}^{\epsilon / k T}\right)}\right]=q(1-q)
$$

Combining eqs $4-7$ and $10-12$, the equation of state $(\mathrm{EoS})$ reads

$$
\begin{array}{r}
\frac{p V}{s N k T}=-\frac{\ln (1-y)}{y}-\left(1-\frac{1}{s}\right)+\frac{\alpha}{\gamma}+\frac{\ln (1-\alpha \gamma)}{\gamma y}- \\
\left(\frac{\epsilon}{\gamma k T}\right) \frac{\bar{x}}{y}+\frac{1}{\gamma y}[(\bar{x}-1) \ln (1-\bar{x}-q)+\bar{x} \ln (q-\bar{x})- \\
2 \bar{x} \ln (\bar{x})+2 \ln (1-q)]
\end{array}
$$

Equation 16 can be presented in scaled variables if the reduced variables are introduced as

$$
\tilde{p}=p / p^{*}, \quad \tilde{V}=V / V^{*}, \quad \tilde{T}=T / T^{*}
$$

The scaling parameters $P^{*}, T^{*}$, and $V^{*}$ depend on the molecular parameters according to

$$
V^{*}=s N \mathrm{v}_{0}, \quad T^{*}=\epsilon / \gamma k, \quad p^{*}=\epsilon / \gamma \mathrm{v}_{0}
$$

The reduced EoS reads

$$
\begin{array}{r}
\frac{\tilde{p} \tilde{V}}{\tilde{T}}=-\frac{\ln (1-y)}{y}-\left(1-\frac{1}{s}\right)+\frac{\alpha}{\gamma}+\frac{\ln (1-\alpha y)}{\gamma y}-\frac{\tilde{x}}{y \tilde{T}}+ \\
\frac{1}{\gamma y}[(\bar{x}-1) \ln (1-\bar{x}-q)+\bar{x} \ln (q-\bar{x})- \\
\left.2 \bar{x} \ln \left(\bar{x}_{\overline{\mathbf{x}}}\right)+2 \ln (1-q)\right]
\end{array}
$$

The results obtained by the maximum term approximation will be called the QC or nonrandom mixing (NRM) theory. From this approximation for the partition function several well-known theories can be derived as limiting cases. For example, the Sanchez-Lacombe (SL) lattice fluid ${ }^{5}$ result is recovered if random mixing is assumed and the coordination number approaches infinity $(z \rightarrow \infty)$. The $\mathrm{EoS}$ is given by the single, simple equation

$$
\frac{\tilde{p} \tilde{V}}{\tilde{T}}=-\frac{\ln (1-y)}{y}-\left(1-\frac{1}{s}\right)-\frac{y}{\tilde{T}}
$$

The application of the quasi-chemical approximation to the equation of state has been discussed by several authors, e.g., Panayiotou and Vera ${ }^{18}$ and Kumar, Suter, and Reid. ${ }^{19}$ It should be noted that these authors only considered the influence of the nonrandom distribution of segmental interactions and assumed random mixing of contacts involving vacancies.

Although the nonrandom mixing theory is somewhat more complicated, it will be shown that, in contrast to the random mixing theory, it offers very accurate predictions of the MC simulation results.

The random SL theory and the nonrandom mixing theory will be applied in the comparison with the simulation results for athermal chains and chains including attractive interactions. In the case of athermals, the last term in eq 20 , derived from the interaction energy, vanishes. In eq 19 the bracketed and interaction energy terms disappear, leading to a relation between the 
compressibility factor $p V / N k T=s \tilde{p} \tilde{V} / \tilde{T}$ and reduced density $y$.

$$
\frac{\tilde{p} \tilde{V}}{\tilde{T}}=-\frac{\ln (1-y)}{y}-\left(1-\frac{1}{s}\right)+\frac{\alpha}{\gamma}+\frac{\ln (1-\alpha \gamma)}{\gamma y}
$$

This last equation can be called the Huggins approximation for the equation of state of chain lattice fluids.

\section{Simulation Method}

(1) The simulation method used here is an extension of a previous one used for compressible systems at zero pressure. The original method consists of condensing polymer chains against a solid wall via interactions between chain segments and a short-range adsorption interaction of the wall toward chain segments. ${ }^{20}$ The resulting polymer slab is compressible, where the density of the condensed polymer is balanced by interactions between the chains and a free exchange of voids between the bulk polymer and the free space above. Except introducing compressibility in the system, this method is very suitable also for interfacial studies. A rectangular section of a cubic lattice is used here with 50 sites in the $Z$-direction and 22 sites in the other two directions. The solid wall is placed at the position $Z=1$, and the system of 200-484 (depending on statistics) chains is condensed against this wall. Periodic boundary conditions are effective only in the $X$ and $Y$ directions. Chain lengths $s=30$ are used mostly; for athermal chains we used also $s=20$. The simulation of chains, their movement and nearest-neighbor nonbonded segment-segment interaction $e=-\epsilon / k T$ resembles that of the previous studies. ${ }^{21}$

(2) To exert a finite pressure in the $N p T$ ensemble, we need to make fluctuations of the total volume which are then used in the Metropolis sampling criterium ${ }^{22}$

$$
\exp \left[-\frac{(\epsilon \Delta n+p \Delta V)}{k T}\right]
$$

where $\Delta n$ is the change in the number of interactions introduced by trial moves of chains and $\Delta V$ is the trial total volume change. The sampling criterium used in the lattice simulations is analogous to that of Boyd ${ }^{10}$ adopted for off-lattice simulations. In order to represent the system in absolute units, we use $T=300 \mathrm{~K}$ and have chosen the volume of one lattice site (or $s$-mer segment) to be $v_{0}=$ $0.0001 \mathrm{~m}^{3} / \mathrm{mol}$, which represents closely the value of the molar volume of a polystyrene segment. This choice, however, does not pose any restriction of the generality of our results because it can easily be shown that Metropolis sampling with $\Delta E$ according to the above equation is dependent only on reduced state variables.

A change in the total volume, attempted each 200 chain configurational moves in the system, is realized by building/destroying a solid piston site-by-site with respect to the solid wall at $Z=1$, pressurizing thus the polymer in between. Chains have only excluded-volume interaction with the piston. A trial volume change $\Delta V= \pm v_{0}$ is attempted at the leading piston segment, selecting randomly the sign of the volume change. If the piston is to move in $\left(\Delta V=-v_{0}\right)$, we inspect the site next to the leading segment. If this site is empty, it is filled by a piston segment according to the Metropolis criterion given by eq 22 . If the site is occupied by a chain segment, the attempted move is rejected and the last configuration is counted once more in averaging. If $\Delta V=+v_{0}$ is chosen, the leading piston segment is removed according to the Metropolis rule, with the resulting configuration again sampled in the averaging. It should be noted that a very long equilibration is needed

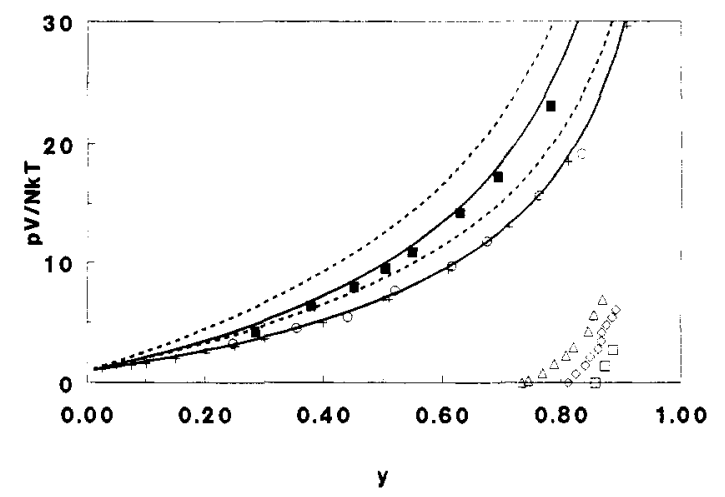

Figure 1. Compressibility factor as a function of filling fraction $y$. (a) Athermal systems: $s=20$; our MC results (O), results of Hertanto and Dickman ${ }^{11}(+) ; s=30(\square)$, respective full lines are obtained by the Huggins approximation (eq 21); dashed lines are by the Flory approximation (eq 20). (b) $s=30 ;-e=0.45(\Delta)$, $0.50(\diamond), 0.55(\square)$.

in the $N p T$ ensemble with entangled polymer chains as analyzed already by Boyd. ${ }^{10}$ The best performance in the simulation is obtained by reading in the configuration from a previous simulation and changing the system parameters by small increments to the new thermodynamic state. We could obtain reliable results up to a filling fraction $y=0.9$. Pressurizing to higher densities was not efficient, in agreement with Hertanto and Dickman's repulsive wall method. ${ }^{11}$ For nonzero pressures we could release the attractive interaction of chain segments with the wall which was present in the original algorithm of Madden. ${ }^{20}$ In this way we studied also the interface at the wall under pressure unperturbed by the interaction with the wall.

\section{Results and Discussion}

a. Bulk Properties. From the simulation data the bulk properties are obtained from the polymer slab showing "bulk" behavior only; i.e., the layer near the wall, the free surface, or piston is not used. In Figure 1 the simulation results on the compressibility factor $p V / N k T$ versus reduced density $y$ both for athermal chains and chains with negative segmental interactions are presented. For comparison, simulation data of Hertanto and Dickman ${ }^{11}$ for athermal 20-mers are included also. The results of both independent simulation studies are in excellent agreement. Since the results of Hertanto and Dickman are obtained with different simulation algorithms (chain insertion and repulsive wall methods), the agreement clearly demonstrates that our simulation method produces accurate and reliable results.

Furthermore, for the athermals the prediction according to the Flory or SL approximation (eq 20) and the Huggins theory (eq 21) are shown. In agreement with previous investigations, the Flory approximation clearly overestimates the compressibility factor $Z$ of the athermal chains due to a too high density over the whole investigated pressure range. On the other hand, the Huggins theory gives an excellent prediction of the data. It has been shown that the Freed theory further improves correspondence at low densities. ${ }^{11}$ However, at the higher densities, which are of practical interest for polymers, the two theories are virtually indistinguishable. Clearly, for relatively short chains the Huggins approximation gives a fair prediction of the equation of state over the complete density range even down to extremely low densities where one would expect deviations with the theory due to the nonhomogeneous segmental density caused by the chain connectivity. Certainly, for the chain lengths considered in this study these effects are not observed. Finally, concluding 


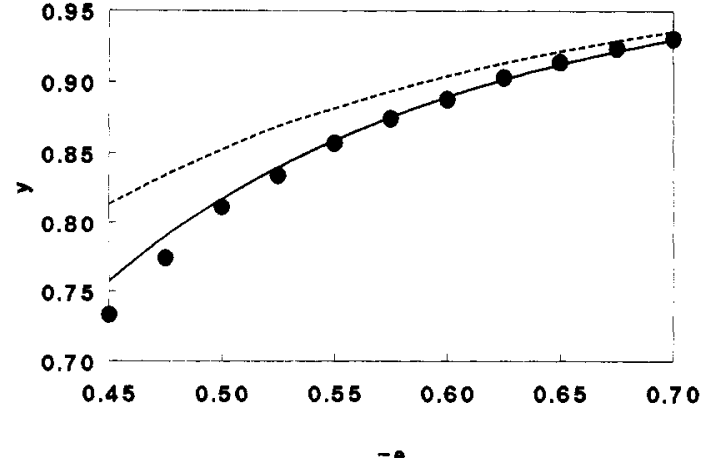

Figure 2. Filling fraction $y$ vs intersegmental interaction $-e$ for $s=30$. (1) Our MC results; the full line represents the NRM prediction and the dashed line the SL theory.

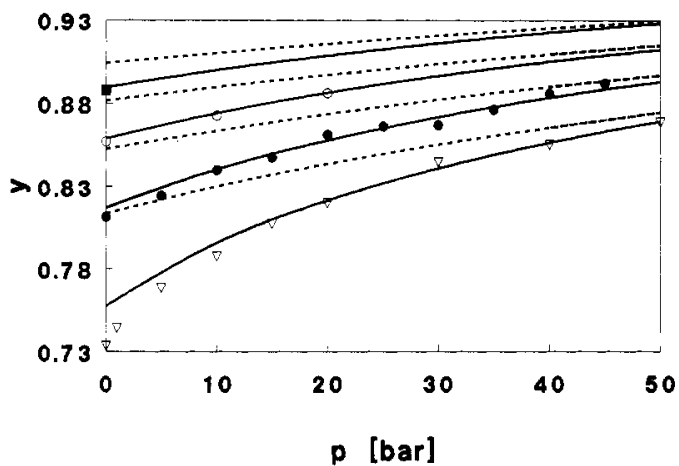

Figure 3. Density as a function of pressure for different interactions: $-e=0.45(\Delta), 0.5(\bullet), 0.55(0), 0.60(\omega)$. Full lines represent the NRM prediction and the dashed lines the SL theory.

the discussion of the athermal chains, deviations between theory and simulation increase for higher chain lengths (compare the results for $s=20$ and $s=30$ in Figure 1). This is also in agreement with previous observations of Hertanto and Dickman. ${ }^{11}$

In the right-hand lower corner the MC results for chains with $s=30$ including interactions are shown to demonstrate how the region of interest is shifted to higher densities and smaller compressibility factors in comparison with the athermal systems. Some sections at constant pressure and temperature are shown in Figures 2 and 3; see also Table 1. In Figure 2 the zero pressure results are shown as a function of reduced interaction energy (or reciprocal reduced temperature). The pressure dependences at a few isothermal conditions are shown in Figure 3. Also in this case the data are compared to the theoretical predictions according to the SL theory (eq 20) and the NRM theory (eq 19). In agreement with the observations made for the athermal chains, the SL theory overestimates the density at all temperatures and pressures, with the smallest deviations at the highest densities, leading to thermal expansion and compressibility coefficients which are too small. However, if the NRM theory is used, quantitative predictions of the complete EoS at densities of practical interest to polymers, i.e., $0.8<y \leq 0.93$, ensue. We expect similar agreement also at higher densities. Apparently, systematic deviations between theory and simulation show up at the highest temperatures and lowest pressures. These deviations are considered in some more detail below.

In contrast to experiment, simulation and theory also provides detailed information concerning the number of nonbonded and segment-hole contacts (the number of nonbonded contacts are directly proportional to the internal energy and are thus also susceptible to experiment). These results are shown in Figures 4 and 5 versus

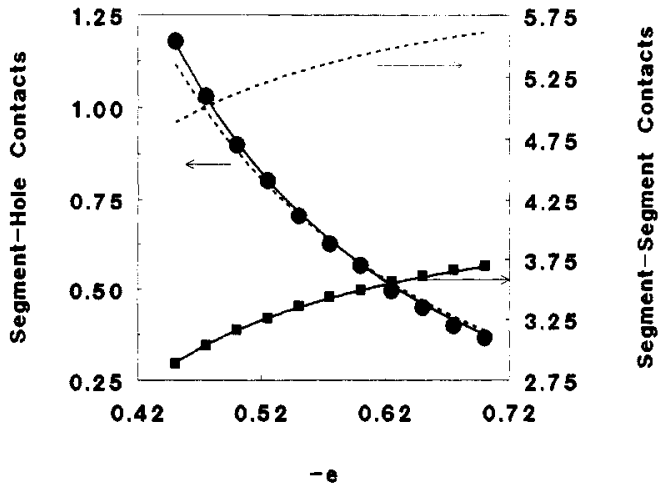

Figure 4. Segmental contacts vs interaction in the melt: (ש) segment-segment contacts; (৩) segment-hole contacts. Full lines represent the NRM theory and dashed lines the SL theory.

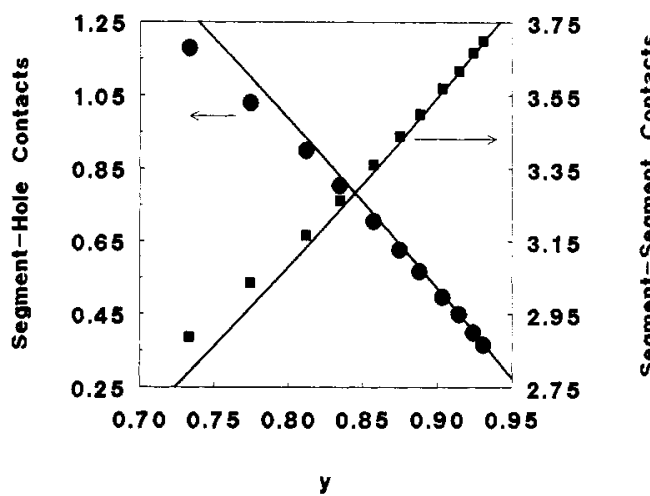

Figure 5. Segmental contacts vs density $y$ corresponding to interactions $e$ in Figure 4. Notation is the same as Figure 4, only prediction of the NRM approach is shown.

Table 1. Monte Carlo Equation of State Results for $T=300 \mathrm{~K}$ and $s=30$

\begin{tabular}{crcc}
\hline$-e$ & $p$ & $y$ & $\begin{array}{c}\text { segment-hole } \\
\text { contacts }\end{array}$ \\
\hline 0.45 & 0 & 0.733 & 1.179 \\
0.45 & 1 & 0.744 & \\
0.45 & 5 & 0.768 & 1.084 \\
0.45 & 10 & 0.788 & 1.014 \\
0.45 & 15 & 0.807 & 0.936 \\
0.45 & 20 & 0.820 & 0.886 \\
0.45 & 30 & 0.845 & 0.781 \\
0.45 & 40 & 0.855 & 0.738 \\
0.45 & 50 & 0.869 & 0.674 \\
0.45 & 75 & 0.894 & \\
0.5 & 0 & 0.812 & 0.901 \\
0.5 & 5 & 0.824 & 0.852 \\
0.5 & 10 & 0.840 & 0.788 \\
0.5 & 15 & 0.848 & 0.757 \\
0.5 & 20 & 0.862 & 0.699 \\
0.5 & 25 & 0.867 & 0.677 \\
0.5 & 30 & 0.867 & 0.673 \\
0.5 & 35 & 0.877 & 0.634 \\
0.5 & 40 & 0.886 & 0.591 \\
0.5 & 45 & 0.892 & 0.564 \\
0.55 & 0 & 0.857 & 0.705 \\
0.55 & 10 & 0.873 & 0.639 \\
0.55 & 20 & 0.886 & 0.581 \\
0.60 & 0 & 0.888 & 0.567 \\
0.65 & 0 & 0.914 & 0.450 \\
0.70 & 0 & 0.931 & 0.366
\end{tabular}

temperature and density, respectively. Especially the SL prediction of the number of segment-segment contacts is poor. The SL theory does not include properly the presence of the covalent bonds between chain segments. This result in a too high value of the segment-segment contacts in Figure 4. Clearly, subtracting the number of bonded contacts from the theoretical lines in Figure 4 would result in better agreement with the simulation data. 


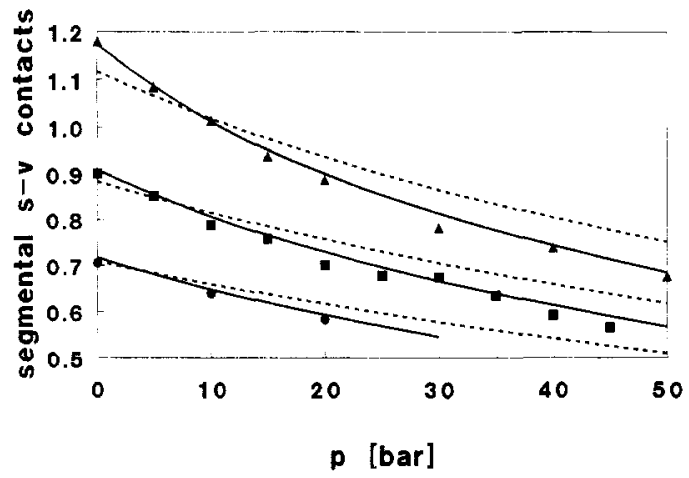

Figure 6. Segment-hold contacts vs pressure for $-e=0.45(\bullet)$, $0.5(\mathbf{\square})$, and $0.55(\mathbf{\Delta})$.

However, this introduces a certain inconsistency since the effect of the nonbonded contacts is only accounted for in the comparison and not in the theoretical calculation. Accounting for the bonded contacts in the calculation, also a less satisfying agreement between simulation and theory is found. The NRM theory provides a quantitative prediction of both types of contact. Surprisingly the deviations discernible in the EoS results are not revealed in the number of contacts. However, these deviations show up again if the contacts are presented as a function of density; see Figure 5. In Figure 6 the segment-vacancy contacts are shown as a function of pressure at the indicated reduced temperatures. Also in this case quantitative agreement is obtained with the NRM theory. For completeness the SL results are shown also.

It is clearly shown that the NRM theory offers a superior prediction of the simulation results, implying that the NMR approach does a better job in evaluating the partition function. From this point of view it seems worthwhile to use the somewhat more complicated NRM equations. However, a direct comparison of the SL and NRM theories with experimental data does not show the same significant improvement. In fact the simple SL theory apparently performs slightly better. However, this is due to the limitations of the molecular model used and is not related to the more sophisticated evaluation of the partition function.

In favor of this conclusion, the Simha-Somcynsky theory ${ }^{2}$ and the more recently devised Holey Huggins ${ }^{8}$ and nonrandom mixing hole or cell fluid ${ }^{9}$ theories indicate that the agreement between theory and experiment systematically improves upon introducing the successive refinements defined by the Huggins correction and the NRM quasi-chemical approximation. These more sophisticated theories perform better compared to the simple lattice theories because the importance of the cell partition function is recognized. ${ }^{1}$

Apparently in Figure 2 the deviations between the NRM theory and simulation results set in at low densities and low pressures. These deviations can be due to (i) the proximity of the vapor-liquid two-phase equilibrium and/ or (ii) the presence of a spatially inhomogeneous segmental density in the fluid at these low densities. Although the mean-field lattice theories presented so far produce incorrect critical scaling results, ${ }^{23}$ a NRM estimate of the location of the vapor-liquid conditions can be made: a $\mathrm{V}-\mathrm{L}$ critical point is predicted $\left(e_{\mathrm{c}}=-0.3111, p_{\mathrm{c}}=0.006\right.$ bar, $y_{c}=0.195$ ), and for even lower pressures a two-phase region develops. This implies that the zero pressure MC results must be situated in the two-phase region, more particularly in the metastable part. At sufficiently high density, i.e., at the lowest temperatures, $\mathrm{MC}$ results practically coincide with the liquid branch of the coexist-

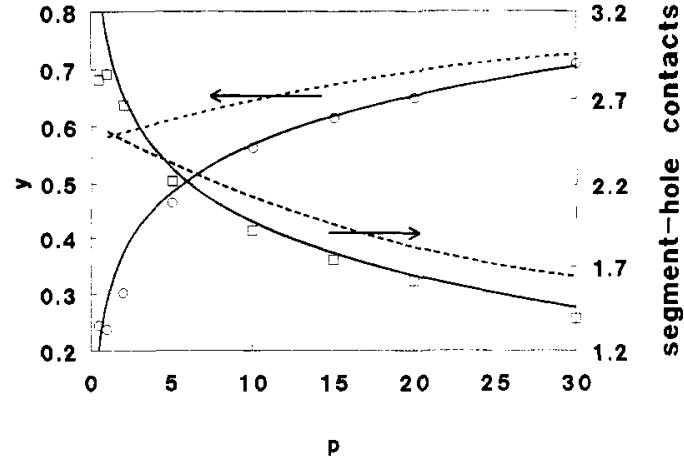

Figure 7. Segment-hole contacts $(\square)$ and density $y(0)$ vs pressure for $-e=0.3$. Full lines represent the NRM theory and dashed lines the SL theory.

ence curve. This makes it possible to identify these data for all practical purposes with the liquid branch of the two-phase region. However, along the V-L coexistence curve at higher temperatures, pressure increases also. Furthermore, the proximity of the critical or spinodal curve in this case may become important and lead to deviations between predicted (mean-field) and simulated densities.

In order to investigate the influence of low densities, simulations were performed at high temperature (or equivalently at low reduced interaction energy) and low pressure while assuring that the system is in the one-phase region of the phase diagram. The simulation results are shown in comparison to the NRM predictions in Figure 7. Unexpectedly, the NRM theory produces accurate predictions of the EoS even up to the lowest density investigated $(y \sim 0.25)$. The largest deviations show up at the intermediate densities $(0.3<y<0.5)$. It should be noted that for the chain length $s=30$ at densities $y<0.35$ isolated coils are present in the system and certainly a spatially inhomogeneous segmental density exists. Nevertheless, the segment-vacancy contacts are predicted accurately although systematic deviations occur at the lowest densities. This agrees with the idea that for these conditions the theory becomes less accurate due to the inhomogeneous segmental density. However, it is known that the intramolecular and intermolecular contributions to the segmental contacts are not properly modeled. For comparison, the predictions according to the SL theory are shown, clearly exposing that the random mixing theory is not capable of predicting the simulation results. Deviations already occurring at high densities are even more pronounced at these low densities, demonstrating the inappropriateness of the random mixing approximation.

b. Situation at the Interface Polymer/Solid Wall. The above-presented analysis was aimed at the bulk EoS properties. Hence, the bulk filling fraction $y$ was not dependent on the $Z$-direction. In this section we analyze the melt profiles $y(Z)$ near the fixed wall, located at $Z=$ 1 , for different pressures and zero interaction of chain segments with the wall. Previous analysis of polymer profiles at a solid wall was mostly performed on athermal and/or semidilute systems. Here we present data also for a condensed polymer with cohesive interaction.

Profiles of athermal systems (Figure 8 ) do not show pronounced changes at the wall. There is only a narrow depletion layer of lower density. In contrast, the systems with interactions exhibit pronounced effect at the interface; see Figure 9. While the pressure affects the bulk density only moderately, it has a huge effect locally at the interface. This is because the diffuse surface of a condensed phase here has a larger compressibility and thus is more susceptible to volume changes. This is well documented by the series of profiles in Figure 9 . 


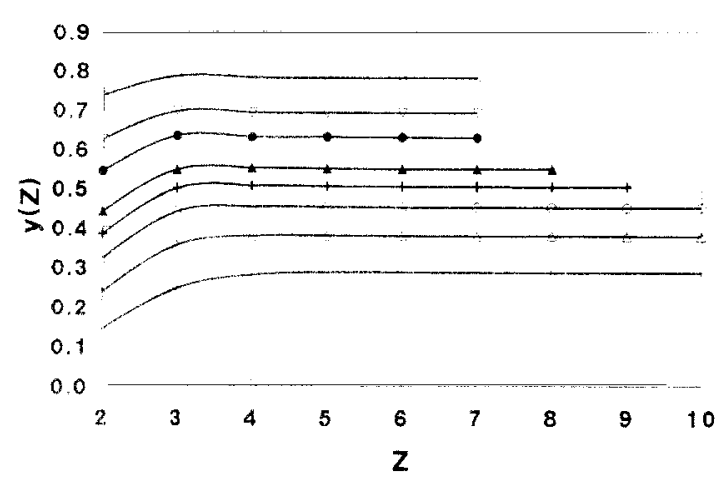

Figure 8. Surface profiles for the athermal system for different pressures $p=10(+), 20(\Delta), 30(0), 40(+), 50(\Delta), 75(\bullet), 100$ $(\nabla)$, and $150(\diamond)$. Solid lines are drawn for convenience.

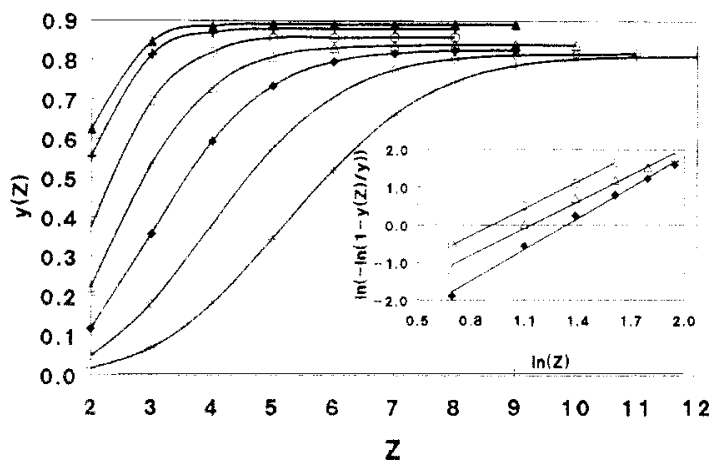

Figure 9. Surface profiles for $e=-0.5$ and different pressures $p=1(+), 2(\diamond), 5(\diamond), 10(\Delta), 20(0), 40(+)$, and $50(\Delta)$. The inset shows scaling analysis by the Shih-Aksay function for $p=$ 5,10 , and 20 . Solid lines are drawn for convenience.

For higher pressures it is observed (Figure 8) that the profile is described by the function used for athermal semidilute systems by Shih, Shih, and Aksay. ${ }^{24}$

$$
y(Z)=y\left(1-\exp \left[-(Z / \zeta)^{m}\right]\right)
$$

with $\zeta$ a correlation length.

As we mentioned, due to relatively short chains in the simulation, the scaling analysis does not provide the predicted exponent $m=1.6$. Our value of the exponent 2.4-2.8 reflects a steeper interface profile than that for a high polymer surface. The other reason for this deviation might be that the function was obtained for the semidilute regime and not the condensed phase.

For low pressures the condensed phase is driven away from the wall by the elasticity of the surface. The elasticity of the interface with the wall ensues from the deformation of the coils near the wall. For the finite pressure simulations this deformation leads to a density depletion near the wall. Consequently, almost the total surface profile, similar to that of the free surface, is shown and is well described by the usual interface profile function ${ }^{25}$

$$
y(Z)=\frac{y}{2}\left[1+\tanh \left(\frac{2(Z-d)}{D}\right)\right]
$$

where $d$ and $D$ are the position and the thickness or full width of the interface. The transition between these two different regimes of behavior, described by eqs 23 and 24 , is well documented by a series of states in between; see Figure 9.

We tested also the conjecture ${ }^{23}$ that the surface density at the wall varies linearly with pressure in the system. Negative deviations from linearity were found (Figure 10) both for $e=0$ and $e=-0.5$ similarly as observed also by Hertanto and Dickman. ${ }^{11}$ It is clear from the figure that

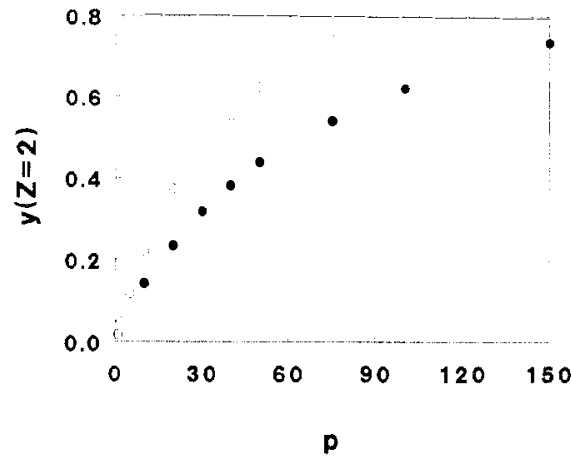

Figure 10. Surface concentration $y(Z=2)$ of the nearest to the wall polymer segment layer vs pressure for $-e=0(0)$ and $0.5(\bullet)$.

for athermal systems a smaller pressure than that for systems with cohesion is needed to build the same surface concentration, indicating again the stronger surface effects in thermal systems.

\section{Conclusions}

The MC simulation data set for athermals and lattice chains with interactions already available in the literature ${ }^{11,20}$ have been extended. For the chains with interactions at zero pressure and the first nonzero pressure results are presented. The simulation results of the bulk properties of interacting lattice chains are accurately predicted by the quasi-chemical or nonrandom mixing theory for the density region of interest to polymeric fluids. The theory which assumes randomness is not able to predict or describe the simulation data consistently. Although the nonrandomness amounts only to approximately $5 \%$ in the number of contacts, they have a rather large influence on the EoS. The deviations in the predicted and simulated equation of state at zero pressure and the highest temperatures are likely related to the proximity of the vapor-liquid critical state. In this region the nonrandom mixing approximation is not sufficient and the mean-field approximations are incorrect.

In the study of the interfacial properties large effects of pressure were observed for the interface with the wall in systems with cohesive interactions in contrast to smaller effects observed for athermal systems. As analyzed, this is related to a broader interface with a variation in density for thermal systems. This conclusion can be extended tentatively to other interfaces with density variation such as the free surface and the polymer/polymer interface with density depletion at the interface. For thermal systems two different types of profiles were observed for low and moderate pressure.

The hard piston method leading to interfaces in only one direction, instead of moving periodic boundaries with concomitant chain configuration adjustment in a homogeneous system, as in the method of Boyd, ${ }^{10}$ allows us to study the interfaces under pressure. The method is not restricted to the interface at the wall but also internal polymer/polymer interfaces can be studied. A compressible layered polymer slab created by the method of Madden ${ }^{20}$ can be placed under the piston and pressurized in order to study the polymer/polymer interface under the pressure. We intend to use this property in our future work.

\section{References and Notes}

(1) Prigogine, I.; Bellemans, A.; Mathot, V. The Molecular Theory of Solutions; North Holland Publishing Co.: Amsterdam, The Netherlands, 1957.

(2) Simha, R.; Somcynsky, T. Macromolecules 1969, 2, 342. 
(3) Flory, P. J.; Orwoll, R. A.; Vrij, A. J. Am. Chem. Soc. 1964, 68, 3567.

(4) Kleintjens, L. A.; Koningsveld, R. Colloid Polym. Sci. 1980, 258,711 .

(5) Sanchez, I. C.; Lacombe, R. H. J. Phys. Chem. 1976, 80, 2352.

(6) Patterson, D. J. Polym. Sci. C 1968, 16, 3379.

(7) Kammer, H. W.; Inoue, T.; Ougizawa, T. Polymer 1989, $30,888$.

(8) Nies, E.; Stroeks, A. Macromolecules 1990, 23, 4088

(9) Nies, E.; Xie, H. Macromolecules 1993, 26, 1683

(10) Boyd, R. H. Macromolecules 1989, 22, 2477.

(11) Hertanto, A.; Dickman, R. J. Chem. Phys. 1988, 89, 7577.

(12) Dickman, R.; Hall, C. K. J. Chem. Phys. 1988, 89, 3168

(13) McQuarrie, D. A. Statistical Mechanics; Harper \& Row: New York, 1976.

(14) Guggenheim, E. A. Mixtures; Oxford University Press: London, 1952.

(15) Huggins, M. L. Ann. N.Y. Acad. Sci. 1942, 43, 1.

(16) Flory, P. J. J. Chem. Phys. 1942, 10, 51.
(17) Freed, K. F.; Bawendi, M. G. J. Phys. Chem. 1989, 93, 2194.

(18) Panayiotou, C.; Vera, J. H. Fluid Phase Equilib. 1980, 5, 55.

(19) Kumar, S. K.; Suter, U. W.; Reid, R. C. Ind. Eng. Chem. Res. $1987,26,2532$

(20) Madden, W. G.; Pesci, A. I.; Freed, K. F. Macromolecules 1990 , 23,1181 .

(21) Cifra, P.; Karasz, F. K.; MacKnight, W. J.Macromolecules 1992, $25,4895$.

(22) Allen, M. P.; Tildesley, D. J. Computer Simulation in Liquids; Oxford University Press: Oxford, U.K., 1990; p 123.

(23) de Gennes, P.-G.; Scaling Concept in Polymer Physics; Cornell University Press: Ithaca, NY, 1979.

(24) Shih, W. Y.; Shih, W.-H.; Aksay, I. A. Macromolecules 1990, 23, 3291.

(25) Helfand, E. Polymer Compatibility and Incompatibility: Principles and Practice; Solc, K., Ed.; MMI Symposium Series Vol. 2; Harwood: New York, 1982; p 143. 